Gazi University
Journal of Science
$\mathrm{http} / /$ dergipark.gov.tr/gujs

\title{
Scattering Function and The Resolvent of The Impulsive Boundary Value Problem
}

\author{
Elgiz BAIRAMOV ${ }^{10}$, Guler Basak OZNUR ${ }^{2, *}$ \\ ${ }^{1}$ University of Ankara, Faculty of Science, Department of Mathematics, 06100, Ankara, Turkey \\ ${ }^{2}$ University of Gazi, Faculty of Science, Department of Mathematics, 06500, Ankara, Turkey \\ Highlights \\ - The study focused on the scattering solutions of the impulsive boundary value problem. \\ - The properties of the scattering function of ISBVP are examined. \\ - Resolvent operator and asymptotic property of Jost function of this problem are found.
}

\begin{tabular}{l} 
Article Info \\
\hline \\
Received:18 Sep 2020 \\
Accepted:13 Jan 2021 \\
Keywords \\
Boundary value problem \\
Density function \\
Impulsive condition \\
Resolvent operator \\
Spectral parameter
\end{tabular}

\section{INTRODUCTION}

Spectral theory of non-selfadjoint singular Sturm-Liouville operators was initiated by Naimark in 1960 [1]. Naimark investigated a part of the continuous spectrum that distrupted the integrity of spectral singularities [1]. Later, in many studies, the eigenvalues and spectral singularities of the boundary value problem with Sturm-Liouville were investigated [2-5].

Some processes are subject to instant and sharp changes due to external factors. These changes are shortterm changes and are too short to be neglected when compared to the entire duration. Neither differential equations nor difference equations are sufficient to model these processes. A new theory was needed for this. To explain such processes mathematically, equations containing impulsive effects, often called impulsive equations, are used. Many researchers have worked on impulsive boundary value problems lately [6-10]. Because many subjects studied in applied sciences are represented by these equations. Let's give a few examples of them. These are the changes in the speed of the valve cover in the transition from open to closed, the vibrating stroke systems, the state of the solid object passing from a certain liquid density to the other liquid density, deaths in the population, changes in closed market product prices, etc. The first research on impulsive differential equations was done by A.D. Myshkis and V.D. Milman [11]. Samoilenka and Perestyuk also made great contributions to this theory [12-14]. Solutions of first and second order differential equations under different impulsive conditions are discussed and the properties provided by these solutions are examined [15-18]. As is well known, there is the spectral parameter only the differential equation in the classical Sturm-Liouville problems. Differently from other studies, innovation in this study 
includes the density function $\rho$ and spectral parameter is in both boundary condition and differential equation. This gives the problem a different perspective.

Let us introduce Sturm-Liouville operator generated by the equation

$$
-v^{\prime \prime}+q(t) v=\hbar^{2} \rho(t) v, \quad t \in[0,1) \cup(1, \infty),
$$

with boundary condition

$$
-\left(\mu_{1} v(0)-\mu_{2} v^{\prime}(0)\right)=\hbar^{2}\left(\delta_{1} v(0)-\delta_{2} v^{\prime}(0)\right)
$$

and impulsive condition

$$
v\left(1^{+}\right)=\gamma v\left(1^{-}\right), \quad v^{\prime}\left(1^{+}\right)=\zeta v^{\prime}\left(1^{-}\right)
$$

where $\gamma, \zeta, \mu_{i}, \delta_{i}, i=1,2$ are real numbers, $\gamma \zeta \neq 0, \delta_{2} \neq 0, \mu_{1} \delta_{2}-\mu_{2} \delta_{1}>0$, $\hbar$ is a spectral parameter and $q$ is a real valued function satisfying the condition

$$
\begin{gathered}
\int_{0}^{\infty}(1+t)|q(t)| d t<\infty . \\
\rho(t)=\left\{\begin{array}{rc}
\eta^{2} ; & 0 \leq t<1 \\
1 ; & 1 \leq t,
\end{array}\right.
\end{gathered}
$$

$\rho$ is a density function defined as above for $\eta \in(0, \infty), \eta \neq 1$.

Under the condition (4), Equation (1) has the bounded solution satisfying the condition

$\lim _{t \rightarrow \infty} e(t, \hbar) e^{-i \hbar t}=1$,

where

$\hbar \in \overline{\mathbb{C}}_{+}:=\{\hbar \in \mathbb{C}: \operatorname{Im} \hbar \geq 0\}$, with $t \in(0, \infty)$.

$e(t, \hbar)$ has the integral representation

$e(t, \hbar)=e^{i \hbar t}+\int_{t}^{\infty} K(t, s) e^{i \hbar s} d s, \hbar \in \overline{\mathbb{C}}_{+}$,

where $K(t, s)$ is defined by the potential function $q[2,19]$.

The set up of this paper is summarized as follows: In section 2, we first find Jost solution, Jost function and scattering function of impulsive Sturm-Liouville boundary value problem (1)-(3). Later we studied the properties of the scattering function of (1)-(3). We also examined the properties of the scattering function of (1)-(3). In section 3, we describe the set of eigenvalues of this problem. We also obtain the asymptotic equation of the Jost function and resolvent operator of this problem. In section 4, by taking an example, we examine the Jost solution, Jost function and scattering function of ISBVP (1)-(3). Finally, in section 5, we make some conclusions.

\section{THE SCATTERING SOLUTIONS}

It is known that $S\left(t, \hbar^{2}, \eta\right)$ and $C\left(t, \hbar^{2}, \eta\right)$ are the fundamental two solutions of the Equation (1) in the interval $[0,1)$ satisfying the initial conditions

$S\left(0, \hbar^{2}, \eta\right)=0, S^{\prime}\left(0, \hbar^{2}, \eta\right)=1$, 
$C\left(0, \hbar^{2}, \eta\right)=1, C^{\prime}\left(0, \hbar^{2}, \eta\right)=0$

Integral representations of these solutions are as follows

$$
S\left(t, \hbar^{2}, \eta\right)=\frac{\sin \hbar \eta t}{\hbar \eta}+\int_{0}^{t} A(t, s) \frac{\sin \hbar \eta s}{\hbar \eta} d s
$$

and

$$
C\left(t, \hbar^{2}, \eta\right)=\cos \hbar \eta t+\int_{0}^{t} B(t, s) \cos \hbar \eta s d s
$$

where the kernel function $A(t, s)$ and $B(t, s)$ are defined by the potential function $q$ [2]. As is well known that the solutions $S\left(t, \hbar^{2}, \eta\right)$ and $C\left(t, \hbar^{2}, \eta\right)$ are entire functions of $\hbar$ and

$W\left[S\left(t, \hbar^{2}, \eta\right), C\left(t, \hbar^{2}, \eta\right)\right]=-1, \quad \hbar \in \mathbb{C}$,

where $W\left[v_{1}, v_{2}\right]$ is the wronskian of the solutions $v_{1}$ and $v_{2}$ of the Equation (1).

Now we consider the following function by the help of $S\left(t, \hbar^{2}, \eta\right), C\left(t, \hbar^{2}, \eta\right)$ and $e(t, \hbar)$ for $\hbar \in \overline{\mathbb{C}}_{+}$

$E(t, \hbar, \eta)=\left\{\begin{array}{cc}w(\hbar, \eta) S\left(t, \hbar^{2}, \eta\right)+\tau(\hbar, \eta) C\left(t, \hbar^{2}, \eta\right) & ; t \in[0,1) \\ e(t, \hbar) & ; t \in(1, \infty) .\end{array}\right.$

From the impulsive condition (3), we write for $\hbar \in \overline{\mathbb{C}}_{+}$

$w(\hbar, \eta) S\left(1, \hbar^{2}, \eta\right)+\tau(\hbar, \eta) C\left(1, \hbar^{2}, \eta\right)=\frac{1}{\gamma} e(1, \hbar)$

$w(\hbar, \eta) S^{\prime}\left(1, \hbar^{2}, \eta\right)+\tau(\hbar, \eta) C^{\prime}\left(1, \hbar^{2}, \eta\right)=\frac{1}{\zeta} e^{\prime}(1, \hbar)$.

$w(\hbar, \eta)$ and $\tau(\hbar, \eta)$ coefficients are obtained as follows

$w(\hbar, \eta)=\frac{1}{\zeta} C\left(1, \hbar^{2}, \eta\right) e^{\prime}(1, \hbar)-\frac{1}{\gamma} C^{\prime}\left(1, \hbar^{2}, \eta\right) e(1, \hbar)$

and

$$
\tau(\hbar, \eta)=\frac{1}{\gamma} S^{\prime}\left(1, \hbar^{2}, \eta\right) e(1, \hbar)-\frac{1}{\zeta} S\left(1, \hbar^{2}, \eta\right) e^{\prime}(1, \hbar) .
$$

The function $E(t, \hbar, \eta)$ is the Jost solution of (1)-(3). From (2), the Jost function of (1)-(3) is given as

$E(\hbar, \eta)=\left(\mu_{2}+\hbar^{2} \delta_{2}\right) w(\hbar, \eta)-\left(\mu_{1}+\hbar^{2} \delta_{1}\right) \tau(\hbar, \eta)$.

It is analytic in $\mathbb{C}_{+}$and continuous up to the real axis.

It is clear that [5]

$W[e(t, \hbar), e(t,-\hbar)]=-2 i \hbar, \quad \hbar \in \mathbb{R} \backslash\{0\}$.

For $\hbar \in \mathbb{R} \backslash\{0\}$, let us consider the other solution of ISBVP (1)-(3) 
$F(t, \hbar, \eta)=\left\{\begin{array}{cl}\psi(t, \hbar, \eta) & ; t \in[0,1) \\ l(\hbar, \eta) e(t, \hbar)+\Phi(\hbar, \eta) e(t,-\hbar) & ; t \in(1, \infty)\end{array}\right.$

where $\psi(t, \hbar, \eta)$ is the solution of (1) given by

$\psi(t, \hbar, \eta)=\left(\mu_{1}+\hbar^{2} \delta_{1}\right) S\left(t, \hbar^{2}, \eta\right)+\left(\mu_{2}+\hbar^{2} \delta_{2}\right) C\left(t, \hbar^{2}, \eta\right)$.

It is easy to see that the function $\psi(t, \hbar, \eta)$ is an entire function with respect to $\hbar$. From (3), we obtain that

$\iota(\hbar, \eta)=\frac{1}{2 i \hbar}\left[\zeta \psi^{\prime}(1, \hbar, \eta) e(1,-\hbar)-\gamma \psi(1, \hbar, \eta) e^{\prime}(1,-\hbar)\right]$

$\Phi(\hbar, \eta)=\frac{1}{2 i \hbar}\left[\gamma \psi(1, \hbar, \eta) e^{\prime}(1, \hbar)-\zeta \psi^{\prime}(1, \hbar, \eta) e(1, \hbar)\right]$.

By using (9), (10), (12) and (13), we find following equations

$l(\hbar, \eta)=-\frac{\gamma \zeta}{2 i \hbar}\left[\overline{w(\hbar, \eta)}\left(\mu_{2}+\hbar^{2} \delta_{2}\right)-\overline{\tau(\hbar, \eta)}\left(\mu_{1}+\hbar^{2} \delta_{1}\right)\right]$

$\Phi(\hbar, \eta)=\frac{\gamma \zeta}{2 i \hbar}\left[w(\hbar, \eta)\left(\mu_{2}+\hbar^{2} \delta_{2}\right)-\tau(\hbar, \eta)\left(\mu_{1}+\hbar^{2} \delta_{1}\right)\right]$

Theorem 1. For all $\hbar \in \mathbb{R} \backslash\{0\}, E(\hbar, \eta) \neq 0$.

Proof. Suppose that there exists a $\hbar_{0}$ in $\mathbb{R} \backslash\{0\}$ such that $E\left(\hbar_{0}, \eta\right)=0$. Using (11), (14) and (15), it is clear that $l\left(\hbar_{0}, \eta\right)=\Phi\left(\hbar_{0}, \eta\right)=0$. Then the solution $F\left(t, \hbar_{0}, \eta\right)$ is a trivial solution of (1)-(3) which gives a contradiction, i.e., $E(\hbar, \eta) \neq 0$ for all $\hbar \in \mathbb{R} \backslash\{0\}$.

Lemma 1. The wronskian of the solutions $E(t, \hbar, \eta)$ and $F(t, \hbar, \eta)$ is found as

$W[E(t, \hbar, \eta), F(t, \hbar, \eta)]=\left\{\begin{array}{cl}-E(\hbar, \eta) ; & t \in[0,1) \\ -\gamma \zeta E(\hbar, \eta) ; & t \in(1, \infty) .\end{array}\right.$

Proof. If we write the wronskian of $E(t, \hbar, \eta)$ and $F(t, \hbar, \eta)$ for $t \in[0,1)$, we get

$$
\begin{aligned}
& W[E(t, \hbar, \eta), F(t, \hbar, \eta)]=E(t, \hbar, \eta) F^{\prime}(t, \hbar, \eta)-F(t, \hbar, \eta) E^{\prime}(t, \hbar, \eta) \\
& =-\left[\left(\mu_{2}+\hbar^{2} \delta_{2}\right) w(\hbar, \eta)-\left(\mu_{1}+\hbar^{2} \delta_{1}\right) \tau(\hbar, \eta)\right] \\
& =-E(\hbar, \eta) .
\end{aligned}
$$

Similarly, for $t \in(1, \infty)$, it is clear that

$W[E(t, \hbar, \eta), F(t, \hbar, \eta)]=-2 i \hbar \Phi(\hbar, \eta)$.

Using (15), we find that

$W[E(t, \hbar, \eta), F(t, \hbar, \eta)]=-\gamma \zeta E(\hbar, \eta)$,

for $t \in(1, \infty)$. This completes the proof.

The scattering function of (1)-(3) is expressed as follows 
$S(\hbar, \eta)=\frac{\overline{E(\hbar, \eta)}}{E(\hbar, \eta)}, \quad \hbar \in \mathbb{R} \backslash\{0\}$

From (11), we write following equality

$S(\hbar, \eta)=\frac{\left(\mu_{2}+\hbar^{2} \delta_{2}\right) \overline{w(\hbar, \eta)}-\left(\mu_{1}+\hbar^{2} \delta_{1}\right) \overline{\tau(\hbar, \eta)}}{\left(\mu_{2}+\hbar^{2} \delta_{2}\right) w(\hbar, \eta)-\left(\mu_{1}+\hbar^{2} \delta_{1}\right) \tau(\hbar, \eta)}$,

for all $\hbar \in \mathbb{R} \backslash\{0\}$.

Theorem 2. For all $\hbar \in \mathbb{R} \backslash\{0\}$, the scattering function satisfies

$\overline{S(\hbar, \eta)}=\mathrm{S}^{-1}(\hbar, \eta)=S(-\hbar, \eta)$

Proof. By using (16), we write

$$
\begin{aligned}
& S(-\hbar, \eta)=\frac{\left(\mu_{2}+\hbar^{2} \delta_{2}\right) \overline{w(-\hbar, \eta)}-\left(\mu_{1}+\hbar^{2} \delta_{1}\right) \overline{\tau(-\hbar, \eta)}}{\left(\mu_{2}+\hbar^{2} \delta_{2}\right) w(-\hbar, \eta)-\left(\mu_{1}+\hbar^{2} \delta_{1}\right) \tau(-\hbar, \eta)} \\
& =\frac{\left(\mu_{2}+\hbar^{2} \delta_{2}\right) w(\hbar, \eta)-\left(\mu_{1}+\hbar^{2} \delta_{1}\right) \tau(\hbar, \eta)}{\left(\mu_{2}+\hbar^{2} \delta_{2}\right) \overline{w(\hbar, \eta)}-\left(\mu_{1}+\hbar^{2} \delta_{1}\right) \overline{\tau(\hbar, \eta)}} .
\end{aligned}
$$

By using (16) and (17), we find that

$\overline{S(\hbar, \eta)}=\mathrm{S}^{-1}(\hbar, \eta)=S(-\hbar, \eta)$

It completes the proof.

\section{EIGENVALUES AND RESOLVENT OPERATOR OF ISBVP}

Let the unlimited solution of the Equation $(1)$ in $(1, \infty)$ be $\check{e}(t, \hbar)$

$\lim _{t \rightarrow \infty} \check{e}(t, \hbar) e^{i \hbar t}=1, \quad \lim _{t \rightarrow \infty} \check{e}^{\prime}(t, \hbar) e^{i \hbar t}=-i \hbar, \quad \hbar \in \overline{\mathbb{C}}_{+}$.

It is known that

$W[e(t, \hbar), \check{e}(t, \hbar)]=-2 i \hbar, \quad t \in(1, \infty), \quad \hbar \in \overline{\mathbb{C}}_{+}$.

Now, let us define the following solutions of (1) for all $\hbar \in \overline{\mathbb{C}}_{+} \backslash\{0\}$ and $\hbar \in \overline{\mathbb{C}}_{-} \backslash\{0\}$

$H_{1}(t, \hbar, \eta)=\left\{\begin{array}{cl}\psi(t, \hbar, \eta) & ; t \in[0,1) \\ \kappa_{1}(\hbar, \eta) e(t, \hbar)+\theta_{1}(\hbar, \eta) \check{e}(t, \hbar) & ; t \in(1, \infty)\end{array}\right.$

and

$H_{2}(t, \hbar, \eta)=\left\{\begin{array}{cl}\psi(t, \hbar, \eta) & ; t \in[0,1) \\ \kappa_{2}(\hbar, \eta) e(t,-\hbar)+\theta_{2}(\hbar, \eta) \check{e}(t,-\hbar) & ; t \in(1, \infty),\end{array}\right.$

respectively. By using (3), we obtain the coefficients for $\hbar \in \overline{\mathbb{C}}_{+} \backslash\{0\}$ 
$\kappa_{1}(\hbar, \eta)=-\frac{1}{2 i \hbar}\left[\gamma \psi(1, \hbar, \eta) \check{e}^{\prime}(1, \hbar)-\zeta \psi^{\prime}(1, \hbar, \eta) \check{e}(1, \hbar)\right]$,

$\theta_{1}(\hbar, \eta)=\frac{1}{2 i \hbar}\left[\gamma \psi(1, \hbar, \eta) e^{\prime}(1, \hbar)-\zeta \psi^{\prime}(1, \hbar, \eta) e(1, \hbar)\right]$

and for $\hbar \in \overline{\mathbb{C}}_{-} \backslash\{0\}$

$\kappa_{2}(\hbar, \eta)=\frac{1}{2 i \hbar}\left[\gamma \psi(1, \hbar, \eta) \check{e}^{\prime}(1,-\hbar)-\zeta \psi^{\prime}(1, \hbar, \eta) \check{e}(1,-\hbar)\right]$,

$\theta_{2}(\hbar, \eta)=-\frac{1}{2 i \hbar}\left[\gamma \psi(1, \hbar, \eta) e^{\prime}(1,-\hbar)-\zeta \psi^{\prime}(1, \hbar, \eta) e(1,-\hbar)\right]$

Using (9) and (10), $\theta_{1}(\hbar, \eta)$ and $\theta_{2}(\hbar, \eta)$ can be written as follows

$\theta_{1}(\hbar, \eta)=\frac{\gamma \zeta}{2 i \hbar}\left[\left(\mu_{2}+\hbar^{2} \delta_{2}\right) w(\hbar, \eta)-\left(\mu_{1}+\hbar^{2} \delta_{1}\right) \tau(\hbar, \eta)\right]$,

$\theta_{2}(\hbar, \eta)=-\frac{\gamma \zeta}{2 i \hbar}\left[\left(\mu_{2}+\hbar^{2} \delta_{2}\right) w(-\hbar, \eta)-\left(\mu_{1}+\hbar^{2} \delta_{1}\right) \tau(-\hbar, \eta)\right]$

respectively.

The solutions $E(t, \hbar, \eta)$ and $H_{1}(t, \hbar, \eta)$ provide for $\hbar \in \overline{\mathbb{C}}_{+} \backslash\{0\}$

$W\left[E(t, \hbar, \eta), H_{1}(t, \hbar, \eta)\right]=\left\{\begin{aligned}-E(\hbar, \eta) ; & t \in[0,1) \\ -\gamma \zeta E(\hbar, \eta) ; & t \in(1, \infty)\end{aligned}\right.$

and $E(t, \hbar, \eta), H_{2}(t, \hbar, \eta)$ satisfy for $\hbar \in \overline{\mathbb{C}}_{-} \backslash\{0\}$

$W\left[E(t, \hbar, \eta), H_{2}(t, \hbar, \eta)\right]=\left\{\begin{aligned}-M(\hbar, \eta) ; & t \in[0,1) \\ -\gamma \zeta M(\hbar, \eta) ; & t \in(1, \infty),\end{aligned}\right.$

where

$M(\hbar, \eta)=\left(\mu_{2}+\hbar^{2} \delta_{2}\right) w(-\hbar, \eta)-\left(\mu_{1}+\hbar^{2} \delta_{1}\right) \tau(-\hbar, \eta)$.

Theorem 3. The Jost function of (1)-(3) satisfies

$$
\begin{aligned}
& E(\hbar, \eta)=\frac{i \hbar^{3} \delta_{2} e^{i \hbar(1-\eta)}}{2 \gamma \zeta}(\gamma+\zeta \eta+o(1)), \quad \hbar \in \overline{\mathbb{C}}_{+}, \quad|\hbar| \rightarrow \infty, \\
& M(\hbar, \eta)=-\frac{i \hbar^{3} \delta_{2} e^{-i \hbar(1-\eta)}}{2 \gamma \zeta}(\gamma+\zeta \eta+o(1)), \quad \hbar \in \overline{\mathbb{C}}_{-}, \quad|\hbar| \rightarrow \infty .
\end{aligned}
$$

Proof. By using (6) and (7), we obtain that

$$
\begin{aligned}
& C\left(1, \hbar^{2}, \eta\right)=e^{-i \hbar \eta}\left(\frac{1}{2}+o(1)\right), \\
& C^{\prime}\left(1, \hbar^{2}, \eta\right)=e^{-i \hbar \eta} \hbar \eta\left(-\frac{i}{2}+O\left(\frac{1}{\hbar}\right)\right)
\end{aligned}
$$

and 
$S\left(1, \hbar^{2}, \eta\right)=\frac{e^{-i \hbar \eta}}{\hbar \eta}\left(\frac{i}{2}+O\left(\frac{1}{\hbar}\right)\right)$

$S^{\prime}\left(1, \hbar^{2}, \eta\right)=e^{-i \hbar \eta}\left(\frac{1}{2}+o(1)\right)$,

for $\hbar \in \overline{\mathbb{C}}_{+},|\hbar| \rightarrow \infty$.

Similarly for $\hbar \in \overline{\mathbb{C}}_{+},|\hbar| \rightarrow \infty$, from (5), we get

$e(1, \hbar)=e^{i \hbar}(1+o(1))$,

$e^{\prime}(1, \hbar)=\hbar e^{i \hbar}\left(i+O\left(\frac{1}{\hbar}\right)\right)$.

Using (25), (26) and (27), we find the asymptotic representation of $E(\hbar, \eta)$. Similarly, equation asymptotic for $M(\hbar, \eta)$ by given (24) can be easily obtained.

Corollary 1. The set of eigenvalues of ISBVP (1)-(3) is

$\sigma_{d}=\left\{\hbar \in \mathbb{C}_{+}: \theta_{1}(\hbar, \eta)=0\right\} \cup\left\{\hbar \in \mathbb{C}_{-}: \theta_{2}(\hbar, \eta)=0\right\}$.

Proof. It is obvious from (18) that the first part of $H_{1}(t, \hbar, \eta)$ in $L_{2}(0,1)$. Besides, if $\theta_{1}(\hbar, \eta)=0$, then the second part of the $H_{1}(t, \hbar, \eta)$ is in $L_{2}(1, \infty)$. Similarly, we find the second part of $\sigma_{d}$. It follows from (11), (20), (21), (22) and the definition of eigenvalues that [20]

$\sigma_{d}=\left\{\hbar \in \mathbb{C}_{+}: \theta_{1}(\hbar, \eta)=0\right\} \cup\left\{\hbar \in \mathbb{C}_{-}: \theta_{2}(\hbar, \eta)=0\right\}$

or

$\sigma_{d}=\left\{\hbar \in \mathbb{C}_{+}: E(\hbar, \eta)=0\right\} \cup\left\{\hbar \in \mathbb{C}_{-}: M(\hbar, \eta)=0\right\}$

Theorem 4. Under condition (4),

$R_{h} f=\int_{0}^{\infty} G(t, s ; \hbar, \eta) f(t) d t$

is the resolvent operator of (1)-(3),

$G(t, s ; \hbar, \eta)= \begin{cases}G_{1}(t, s ; \hbar, \eta) ; & \hbar \in \overline{\mathbb{C}}_{+} \\ G_{2}(t, s ; \hbar, \eta) ; & \hbar \in \overline{\mathbb{C}}_{-}\end{cases}$

and

$G_{1}(t, s ; \hbar, \eta)= \begin{cases}\frac{E(t, \hbar, \eta) H_{1}(s, \hbar, \eta)}{W\left[E(t, \hbar, \eta), H_{1}(t, \hbar, \eta)\right]} ; 0 \leq s<t \\ \frac{H_{1}(t, \hbar, \eta) E(s, \hbar, \eta)}{W\left[E(t, \hbar, \eta), H_{1}(t, \hbar, \eta)\right]} ; t \leq s<\infty,\end{cases}$ 
$G_{2}(t, s ; \hbar, \eta)= \begin{cases}\frac{E(t,-\hbar, \eta) H_{2}(s, \hbar, \eta)}{W\left[E(t,-\hbar, \eta), H_{2}(t, \hbar, \eta)\right]} ; & 0 \leq s<t \\ \frac{H_{2}(t, \hbar, \eta) E(s,-\hbar, \eta)}{W\left[E(t,-\hbar, \eta), H_{2}(t, \hbar, \eta)\right]} ; & t \leq s<\infty,\end{cases}$

is defined as Green function for all $t \neq 1, s \neq 1$.

Proof. In order to get resolvent operator, we will consider the following equation

$-v^{\prime \prime}+q(t) v-\hbar^{2} v=f(t), \quad t \in[0,1) \cup(1, \infty)$

By using (8) and (18), we write the solution of (28)

$\varphi(t, \hbar, \eta)=d_{1}(t, \hbar) E(t, \hbar, \eta)+d_{2}(t, \hbar) H_{1}(t, \hbar, \eta)$.

Using the method of variation of parameters, $d_{1}(t, \hbar)$ and $d_{2}(t, \hbar)$ are found as follows

$d_{1}(t, \hbar)=a+\int_{0}^{t} \frac{f(s) H_{1}(s, \hbar, \eta)}{W\left[E(t, \hbar, \eta), H_{1}(t, \hbar, \eta)\right]} d s$,

$d_{2}(t, \hbar)=b+\int_{t}^{\infty} \frac{f(s) E(s, \hbar, \eta)}{W\left[E(t, \hbar, \eta), H_{1}(t, \hbar, \eta)\right]} d s$,

where $a$ and $b$ are real numbers. It is obvious that the solution $\varphi(t, \hbar, \eta)$ in $L_{2}(0, \infty)$. Then we find that $b=0$. From (2), we write the following equation

$a\left[\left(\mu_{1}+\hbar^{2} \delta_{1}\right) \tau(\hbar, \eta)-\left(\mu_{2}+\hbar^{2} \delta_{2}\right) w(\hbar, \eta)\right]=0$.

By using (10) and Theorem 1, we get that $a=0$. So we clearly write that

$\varphi(t, \hbar, \eta)=E(t, \hbar, \eta) \int_{0}^{t} \frac{f(s) H_{1}(s, \hbar, \eta)}{W\left[E(t, \hbar, \eta), H_{1}(t, \hbar, \eta)\right]} d s+H_{1}(t, \hbar, \eta) \int_{t}^{\infty} \frac{f(s) E(s, \hbar, \eta)}{W\left[E(t, \hbar, \eta), H_{1}(t, \hbar, \eta)\right]} d s$.

The second part is obtained similarly. It completes the proof.

\section{UNPERTURBATED IMPULSIVE EQUATIONS}

In this section, we give a special example as an application to draw attention to the validity of our results. We study Jost solution, Jost function and scattering function of this example.

Example 1. We shall define the following impulsive Sturm-Liouville boundary value problem

$-v^{\prime \prime}=\hbar^{2} \rho(t) v, \quad t \in[0,1) \cup(1, \infty)$,

$-\left(\mu_{1} v(0)-\mu_{2} v^{\prime}(0)\right)=\hbar^{2}\left(\delta_{1} v(0)-\delta_{2} v^{\prime}(0)\right)$,

$v\left(1^{+}\right)=v\left(1^{-}\right), \quad v^{\prime}\left(1^{+}\right)=v^{\prime}\left(1^{-}\right)$,

where $\mu_{i}, \delta_{i}, i=0,1$ are real numbers, $\mu_{1} \delta_{2}-\mu_{2} \delta_{1}>0$, $\hbar$ is a spectral parameter and $\rho$ is a density function and it is defined as follows

$\rho(t)=\left\{\begin{array}{rc}\eta^{2} ; & 0 \leq t<1 \\ 1 ; & 1 \leq t\end{array}\right.$ 
where $\eta \in(0, \infty), \eta \neq 1$. It is evident that

$e(t, \hbar)=e^{i \hbar t}, \quad S\left(t, \hbar^{2}, \eta\right)=\frac{\sin \hbar \eta t}{\hbar \eta}, \quad C\left(t, \hbar^{2}, \eta\right)=\cos \hbar \eta t$,

for this problem. By using the solutions $e(t, \hbar), S\left(t, \hbar^{2}, \eta\right)$ and $C\left(t, \hbar^{2}, \eta\right)$, we get

$E(t, \hbar, \eta)=\left\{\begin{array}{cc}m(\hbar, \eta) \frac{\sin \hbar \eta t}{\hbar \eta}+r(\hbar, \eta) \cos \hbar \eta t & ; t \in[0,1) \\ e^{i \hbar t} & ; t \in(1, \infty),\end{array}\right.$

where

$m(\hbar, \eta)=e^{i \hbar}(\hbar \eta \sin \hbar \eta+i \hbar \cos \hbar \eta), \quad r(\hbar, \eta)=e^{i \hbar}\left(\cos \hbar \eta-i \frac{\sin \hbar \eta}{\eta}\right)$.

The function $E(t, \hbar, \eta)$ is called Jost solution of ISBVP (29). Using (30), we obtain the Jost function of (29)

$E(\hbar, \eta)=\left(\mu_{2}+\hbar^{2} \delta_{2}\right) m(\hbar, \eta)-\left(\mu_{1}+\hbar^{2} \delta_{1}\right) r(\hbar, \eta), \quad \hbar \in \overline{\mathbb{C}}_{+}$

and scattering function of (29) for $\hbar \in \mathbb{R} \backslash\{0\}$

$$
S(\hbar, \mathrm{\eta})=e^{-2 i \hbar}\left(\frac{\left(\mu_{2}+\hbar^{2} \delta_{2}\right)[(\hbar \eta \sin \hbar \eta-i \hbar \cos \hbar \eta)]-\left(\mu_{1}+\hbar^{2} \delta_{1}\right)\left[\cos \hbar \eta+i \frac{\sin \hbar \eta}{\eta}\right]}{\left(\mu_{2}+\hbar^{2} \delta_{2}\right)[(\hbar \eta \sin \hbar \eta+i \hbar \cos \hbar \eta)]-\left(\mu_{1}+\hbar^{2} \delta_{1}\right)\left[\cos \hbar \eta-i \frac{\sin \hbar \eta}{\eta}\right]}\right) .
$$

\section{CONCLUSIONS}

This paper is devoted to Sturm-Liouville boundary value problem with an impulsive condition. Even though there are various studies devoted to the investigation of scattering analysis of boundary value problems, only a few of them are related to scattering analysis of impulsive boundary value problems. In this paper, we examine the spectral properties of impulsive Sturm-Liouville Equations (1)-(3). Firstly, we give information about the scattering solutions of ISBVP (1)-(3). By the help of these scattering solutions, we obtain the Jost function and scattering function of the problem. After, we find discrete spectrum and resolvent operator of (1)-(3). Unlike other studies, the innovation of this study is that the Equation (1) involves the density function $\rho$. Furthermore, the spectral parameter exists in both differential equation and boundary condition. The study offers a different perspective for researchers working on scattering theory.

\section{CONFLICTS OF INTEREST}

No conflict of interest was declared by the authors.

\section{REFERENCES}

[1] Naimark, M. A., "Investigation of the spectrum and the expansion in eigenfunctions of a nonself adjoint differential operators of the second order on a semi axis", American Mathematical Society Translations, 16(2): 103-193, (1960).

[2] Levitan, B. M., Sargsjan, I. J., "Sturm-Liouville and Dirac operators", Kluwer Academic Publishers Group, Dordrecht, (1991). 
[3] Schwartz, J., "Some non-self adjoint operators", Communications on Pure and Applied Mathematics, 13: 609-639, (1960).

[4] Pavlov, B. S., "On the spectral theoy of non-self-adjoint differential operators", Doklady Akademii Nauk: SSSR., 146: 1267-1270, (1962).

[5] Guseinov, G. S., "On the concept of spectral singularities", Pramana Journal of Physics, 73(3): 587-603, (2009).

[6] Allahverdiev, B. P., Bairamov, E., Ugurlu, E., "Eigenparameter dependent Sturm-Liouville problems in boundary conditions with transmission conditions", Journal of Mathematical Analysis and Applications, 401(1): 388-396, (2013).

[7] Bairamov, E., Aygar, Y., Eren B., "Scattering theory of impulsive Sturm-Liouville equations", Filomat, 31(17): 5401-5409, (2017).

[8] Guseinov, G. S., "Boundary value problems for nonlinear impulsive Hamilton systems”, Journal of Computational and Applied Mathematics, 259: 780-789, (2014).

[9] Bairamov, E., Aygar Y., Oznur, G. B., "Scattering properties of eigenparameter-dependent impulsive Sturm-Liouville equations", Bulletin of the Malaysian Mathematical Sciences Society, 43: 2769-2781, (2020).

[10] Mukhtarov, F. S., Aydemir, K., Mukhtarov, O.Sh., "Spectral analysis of one boundary valuetransmission problem by means of Green's function", Electronic Journal of Mathematical Analysis and Applications, 2: 23-30, (2014).

[11] Milman, V. D., Myshkis, A. D., "On the stability of motion in the presence of impulses", Siberian Mathematical Journal, 1: 233-237, (1960).

[12] Perestyuk, N. A., Plotnikov, V. A., Samoilenko, A. M., Sikripnik, N. V., "Differential equations with impulse effects: Multivaled right-hand sides with discontinuities", De Gruyter Studies in Mathematics 40, Germany, (2011).

[13] Perestyuk, N. A., Samoilenko, A. M., Stanzhitsii, A.N., "On the existence of periodic solutions of some classes of systems of differential equations with random impulse action", Ukrainian Mathematical Journal, 53: 1061-1079, (2001).

[14] Samoilenka, A. M., Perestyuk, N. A., "Impulsive differential equations", World Scientific, Singapore, (1995).

[15] Ugurlu, E., Bairamov, E., "Spectral analysis of eigenparameter dependent boundary value transmission problems", Journal of Mathematical Analysis and Applications, 413(1): 482-494, (2014).

[16] Lakmeche, A., Arino, O., "Bifurcation of nontrivial periodic solutions of impulsive differential equations arising from chemotherapeutic treatment", Dynamics of Continuous, Discrete and Impulsive Systems Series A: Mathematical Analysis, 7: 265-287, (2000).

[17] Nenov, S. I., "Impulsive controllability and optimization problems in population dynamics", Nonlinear Analysis, 36: 881-890, (1999). 
[18] Kadakal, M., Mukhtarov, O.Sh., Mukhtarov, F. S., "Some spectral properties of Sturm-Liouville problem with transmission conditions", Iranian Journal of Science and Technology Transaction A, Science , 29(2): 229-245, (2005).

[19] Marchenko, V. A., "Sturm-Liouville operators and applications", Birkhauser, Verlag, (1986).

[20] Naimark, M. A., "Linear differential operators. Part II: Linear differential operators in Hilbert space”, World Scientific Publishing Co., Inc., River Edge, (1995). 